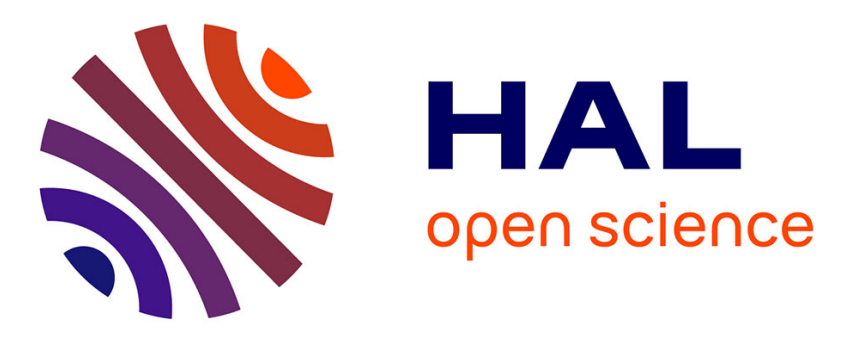

\title{
The effects of filtration on the injection of cement-based grouts in sand columns
}

Olivier Chupin, Nadia Saiyouri, Pierre-Yves Hicher

\section{To cite this version:}

Olivier Chupin, Nadia Saiyouri, Pierre-Yves Hicher. The effects of filtration on the injection of cementbased grouts in sand columns. Transport in Porous Media, 2007, 72 (2), pp.227-240. 10.1007/s11242007-9146-z . hal-01007365

\section{HAL Id: hal-01007365 https://hal.science/hal-01007365}

Submitted on 8 Apr 2017

HAL is a multi-disciplinary open access archive for the deposit and dissemination of scientific research documents, whether they are published or not. The documents may come from teaching and research institutions in France or abroad, or from public or private research centers.
L'archive ouverte pluridisciplinaire HAL, est destinée au dépôt et à la diffusion de documents scientifiques de niveau recherche, publiés ou non, émanant des établissements d'enseignement et de recherche français ou étrangers, des laboratoires publics ou privés. 


\title{
The effects of filtration on the injection of cement-based grouts in sand columns
}

\author{
Olivier Chupin · Nadia Saiyouri • Pierre-Yves Hicher \\ Research Institute in Civil and Mechanical Engineering (GeM), UMR CNRS 6183, Ecole Centrale \\ de Nantes, BP 92101, Nantes cedex 3, France
}

\begin{abstract}
This article presents injection experiments and modeling of cement based grout in sand. In particular, it focuses on the role of filtration during the sand impregnation by the grout. One-dimensional injection tests in sand columns are performed. In these, the mass intake of the sample and the injection pressure are measured to quantify the effects of filtration during grouting. The cement-to-water ratio of the grout and the initial density of the soil are also studied. The modeling of these tests is achieved by incorporating the filtration and the damage coefficients in the classical transport in porous media equations. A method is proposed to determine these coefficients. The method simultaneously relies on both analytical analysis and experimental measurements. Density and viscosity effects are also considered in the model equations which are solved using the finite element method. The simulation of an injection test proves that the model is suitable to recover the injection pressure obtained experimentally. Finally, both experimental and numerical results reveal the importance of including filtration when analyzing one-dimensional injections of cement based grouts in sand.
\end{abstract}

Keywords Grouting · Deep bed filtration · Flow · Transport · Finite element method

\section{Introduction}

This article deals with grouting experiments and modeling. The injection or grouting technique is an engineering process, which consists in injecting grout under pressure into a soil in order to improve its strength or to reduce its permeability. The result of this treatment is obtained after the grout has hardened. This is a traditional process but, for environmental reasons, former chemical grouts have been recently replaced by cement-based ones. By 
nature, those are composed of particles that can be responsible for the appearance of filtration phenomenon during the grout propagation within a soil. Until recently, filtration was not integrated to studies about grouting. Only few papers on this subject are available in the literature (Saada et al. 2005; Bouchelaghem and Vulliet 2001a; Eriksson et al. 2000; Schwarz 1997), and more investigations are needed to better understand the role of this phenomenon during injection.

From a general point of view, different kinds of filtration can be encountered when a fluid transporting particles flows in a porous material. They are enumerated in McDowell-Boyer et al. (1986). However, with the grading curves of the grout and soil used in this article, only deep bed filtration is considered. The latter appears, when the particles of a suspension are small enough to flow through a porous medium. Some of them just keep trapped into the medium under either mechanical or physicochemical forces (Herzig et al. 1970 cited by Dulien 1979), leading to a modification of the flow regime. According to the same authors, the physicochemical and mechanical forces may have simultaneous contributions to filtration for particles whose diameter is in between 1 and $30 \mu \mathrm{m}$.

In this framework, a mathematical model is presented to simulate grouting tests. It is based on the continuum description of a multi-phase system, as detailed in Bear and Bachmat (1991) and Hassanizadeh and Gray (1979). The proposed model depends on two filtration parameters, namely the filtration and the formation damage coefficients (Herzig et al. 1970; Isawaki 1937). Dispersion effects are included through a relation proposed by Bear (1972). Other types of approaches are presented in Tarafdar et al. (1992) and Rege and Fogler (1988). The filtration parameters are assumed to be constant. They are determined by using the analytical solution of the model equations in a simplified case and one-dimensional injection tests. A similar procedure which relies on several pressure drop measurements in a sand sample and an analytical solution of the flow problem is adopted in Bedrikovetsky et al. (2001).

One-dimensional injection tests in sand columns are also exposed in this article. The mass intake of the sample and the injection pressure are measured during those experiments to exhibit filtration effects during grouting. The influence on the tests of the cement-to-water ratio of the grout and the initial density of the soil is also studied. As already mentioned, in addition to providing information about the consequences of filtration during grouting, these tests are used for the determination of the filtration parameters.

Finally, the model equations of the general nonlinear and coupled problem are solved numerically. A code based on the finite element method is developed. It involves many numerical methods to overcome all the challenges inherent to the resolution of coupled flow and transport problems with possible density effects. These are mentioned in Diersch and Kolditz (2002). An injection test is simulated by using the developed code. To assess the ability of the model to predict experimental data, the injection pressure obtained numerically is compared to the one measured during the test.

\section{Mathematical model}

A two phase medium composed of a rigid skeleton and a fluid phase is considered. Initially, the whole pore space is saturated with one fluid. Taking filtration into account, the transport of a suspension miscible with the existing fluid medium is modeled over a time period $J=[0, T]$. The medium is denoted $\Omega \in \mathbb{R}^{d}, d=1,2,3$; it has a smooth boundary $\partial \Omega$. The proposed model is based on the advection-dispersion equation in porous media. 
The advection part is related to the mean flow velocity expressed by the Darcy law

$$
\mathbf{q}=\phi \mathbf{v}^{f}=-\frac{\mathbf{k}}{\mu^{f}}\left(\nabla p-\rho^{f} \mathbf{g}\right), \quad(\mathbf{x}, t) \in \Omega \times J,
$$

where $\mathbf{q}$ is the Darcy velocity and $\mathbf{v}^{f}$ represents the fluid velocity with respect to a fixed coordinate system. Superscript $f$ refers to quantities of the fluid phase. $\mathbf{k}$ is the permeability tensor, $p$ the pore pressure, $\mu^{f}$ the viscosity of the fluid phase, $\rho^{f}$ the density of the fluid phase, $\phi$ the porosity, and $\mathbf{g}$ the gravity. The dispersion effects related to the transported species, including molecular diffusion, are approached through a relation proposed by Bear (1972)

$$
\mathbf{D}=a_{T}|\mathbf{q}| \mathbf{I}+\left(a_{L}-a_{T}\right) \frac{\mathbf{q} \otimes \mathbf{q}}{|\mathbf{q}|}+\phi D_{d} \mathbf{T}, \quad(\mathbf{x}, t) \in \Omega \times J,
$$

where $\mathbf{D}$ is the hydrodynamic dispersion tensor, $a_{L}$ denotes the longitudinal dispersion coefficient and $a_{T}$ the transversal one, $D_{d}$ is the molecular diffusion coefficient and $\mathbf{T}$ is the tortuosity.

Due to filtration, the initial permeability of the medium is physically reduced during the transport of a suspension, and thus modeled by the hyperbolic function (Isawaki 1937) below:

$$
\mathbf{k}(\mathbf{x}, t)=\frac{\mathbf{k}_{0}}{1+\beta \chi}, \quad(\mathbf{x}, t) \in \Omega \times J .
$$

Equation (3) depends on the damage coefficient, $\beta$, and the concentration of the filtrated particles, $\chi$. A similar law is suggested by Saada et al. (2005) for the porosity of the medium in the context of grouting.

The mass balance equation for the transported suspension

$$
\phi \frac{\partial c}{\partial t}+\nabla \cdot(c \mathbf{q})-\nabla \cdot(\mathbf{D} \nabla c)=-\lambda|\mathbf{q}| c, \quad(\mathbf{x}, t) \in \Omega \times J
$$

accounts for mass exchanges between the transported and the filtrated particles via the right hand side term of (4). $c$ denotes the concentration of the transported component (mass of component per unit volume of fluid), and $\lambda$ is the filtration coefficient. The evolution of the concentration of the filtrated component, $\chi$

$$
\phi \frac{\partial \chi}{\partial t}=\lambda|\mathbf{q}| c, \quad(\mathbf{x}, t) \in \Omega \times J,
$$

is proportional to this coefficient, and is expressed as a function of the concentration of the transported component and the mean velocity of the fluid phase.

The flow equation

$$
\nabla \cdot \mathbf{q}=0,(\mathbf{x}, t) \in \Omega \times J,
$$

is obtained from the total mass balance of the whole fluid phase by considering the Boussinesq assumption. The latter consists in neglecting all density dependencies in the balance terms, except for the buoyancy term, $\rho \mathbf{g}$ which is retained in the Darcy equation. The compressibility of the fluid phase is thus neglected in (6). Finally, the evolution laws below:

$$
\rho^{f}=\rho^{f}(\mathbf{x}, t), \quad \mu^{f}=\mu^{f}(\mathbf{x}, t),
$$




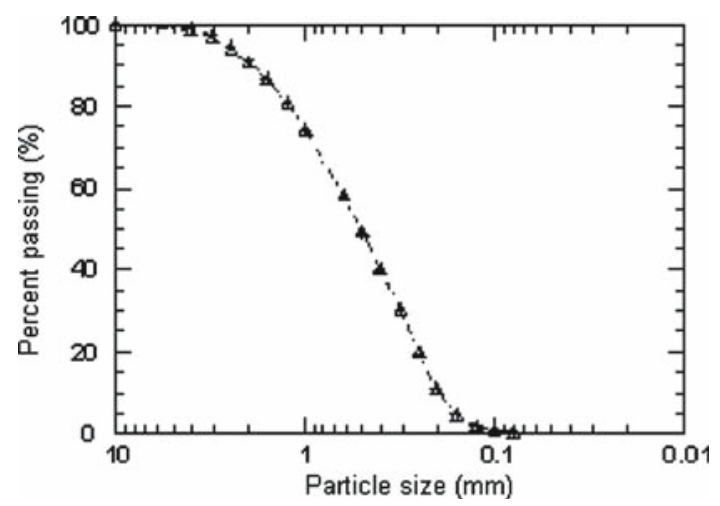

Fig. 1 Grading curve of the Loire river sand

enable to take into account the evolution of the fluid phase properties. The expression of these laws are specified in Sects. 4 and 5.

\section{One-dimensional grouting experiments}

This section provides information about the filtration appearing during the injection of a cement-based grout in a granular material by analyzing the influence of two key parameters: the medium density and the cement-to-water mass ratio $(C / W)$ of the grout. Nowadays, the grouting engineering process is performed by injecting this kind of grout in soils and the filtration induced by the particles in suspension in the grout remains a point to be clarified.

Injection tests are performed at a constant inflow rate from the bottom to the top of a transparent tube of height 1.0 and $0.1 \mathrm{~m}$ in diameter filled with Loire river sand whose properties are given in Dano et al. (2003). This sand is composed of sub-rounded particles and elongated shell fragments. Its grading curve is plotted in Fig. 1.

Typically, during an injection test, the cement particles are intercepted by the porous medium resulting in a transient reduction of the medium permeability. Consequently, as far as a constant inflow rate is maintained, the pore pressure increases even after the grout has saturated the sand column. This is obviously not true for a fluid without particles and the pore pressure can be used to quantify the filtration effects. In order to measure the injection pressure, a transducer is located at the bottom of the column. Moreover, the effluent fluid mass is weighted at the outlet of the tube. The mass intake of the sample also provides indications on the amount of filtrated particles. It is deduced by subtraction of the inlet and the outlet masses of the flowing fluid. The experimental set-up is shown in Fig. 2.

Prior to the grouting phase the sand sample is saturated with water. The filling procedure of the tube must permit to control the global density of the medium. Therefore, the sand is deposited by successive layers of about $0.1 \mathrm{~m}$ whose density is controlled by a number of hammer knocks applied at the surface of the column. The permeability of the resulting sample is measured using a constant-head permeameter. The good repeatability of the filling procedure in terms of permeability measurements proved the reliability of the method.

The patented grout used for the tests is composed of a very-fine cement (Spinor A12), water, a plasticizer additive, and an inert charge. The cement particle diameter is lower than $12 \mu \mathrm{m}$ (Table 1 ) and media with permeability below $10^{-4} \mathrm{~m} / \mathrm{s}$ can be injected using this grout. 


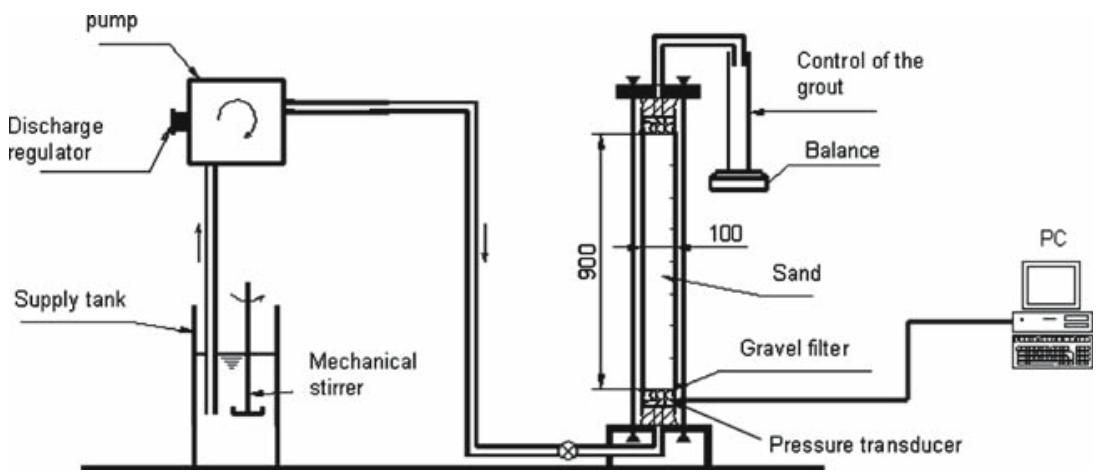

Fig. 2 Experimental set-up for the injection tests

Table 1 Grain sizes of the injected grout

Table 2 Characteristics of the injection tests

\begin{tabular}{lllllll}
\hline Grain size & D15 & D30 & D50 & D60 & D90 & $D_{\max }$ \\
\hline Value $(\mu \mathrm{m})$ & 1 & 2 & 3.5 & 4.5 & 8 & 12 \\
\hline
\end{tabular}

\begin{tabular}{lcccc}
\hline Test & $\begin{array}{c}\text { Initial soil density } \\
\left(\times 1,000 \mathrm{~kg} / \mathrm{m}^{3}\right)\end{array}$ & Porosity & $C / W$ & $\begin{array}{c}\text { Grout density } \\
\left(\times 1,000 \mathrm{~kg} / \mathrm{m}^{3}\right)\end{array}$ \\
\hline Injection11a & 17.22 & 0.34 & 0.28 & 1.36 \\
Injection11b & 17.30 & 0.34 & 0.28 & 1.36 \\
Injection12a & 17.20 & 0.34 & 0.42 & 1.41 \\
Injection12b & 17.24 & 0.34 & 0.42 & 1.41 \\
Injection21a & 18.16 & 0.31 & 0.28 & 1.36 \\
Injection21b & 18.06 & 0.31 & 0.28 & 1.36 \\
Injection22a & 18.14 & 0.31 & 0.42 & 1.41 \\
Injection22b & 18.19 & 0.31 & 0.42 & 1.41 \\
\hline
\end{tabular}

Two granular media with a different porosity are injected by using two grouts of different $C / W$ ratio. Each experiment is conducted twice to ensure the reliability of the results. The test properties are summarized in Table 2.

Figure 3 shows the evolution of the injection pressure and the mass intake with respect to time for each test. As mentioned by Saada et al. (2005), the mass intake of the sample exhibits two distinct kinetic phases (Fig. 3a, c, e, g). The first one lasts up to few seconds after the saturation time $\left(t_{\text {sat }}\right)$ which corresponds to the grout arrival in the outlet flow. During this phase, the grout is progressively mixing in the water that initially saturated the pore space. The second phase $\left(t>t_{\text {sat }}\right)$ is due to filtration only and results from the continuous capture of grout particles by the porous medium. It shows a lower rate than the first phase. For the comparison of the different curves (pressure and mass intake) in terms of filtration we are interested in their second phase appearing when the pore space is entirely filled up with grout $\left(t>t_{\text {sat }}\right)$. From this time, a fluid without particles would reach a permanent flow regime with no more pressure or mass evolution. 

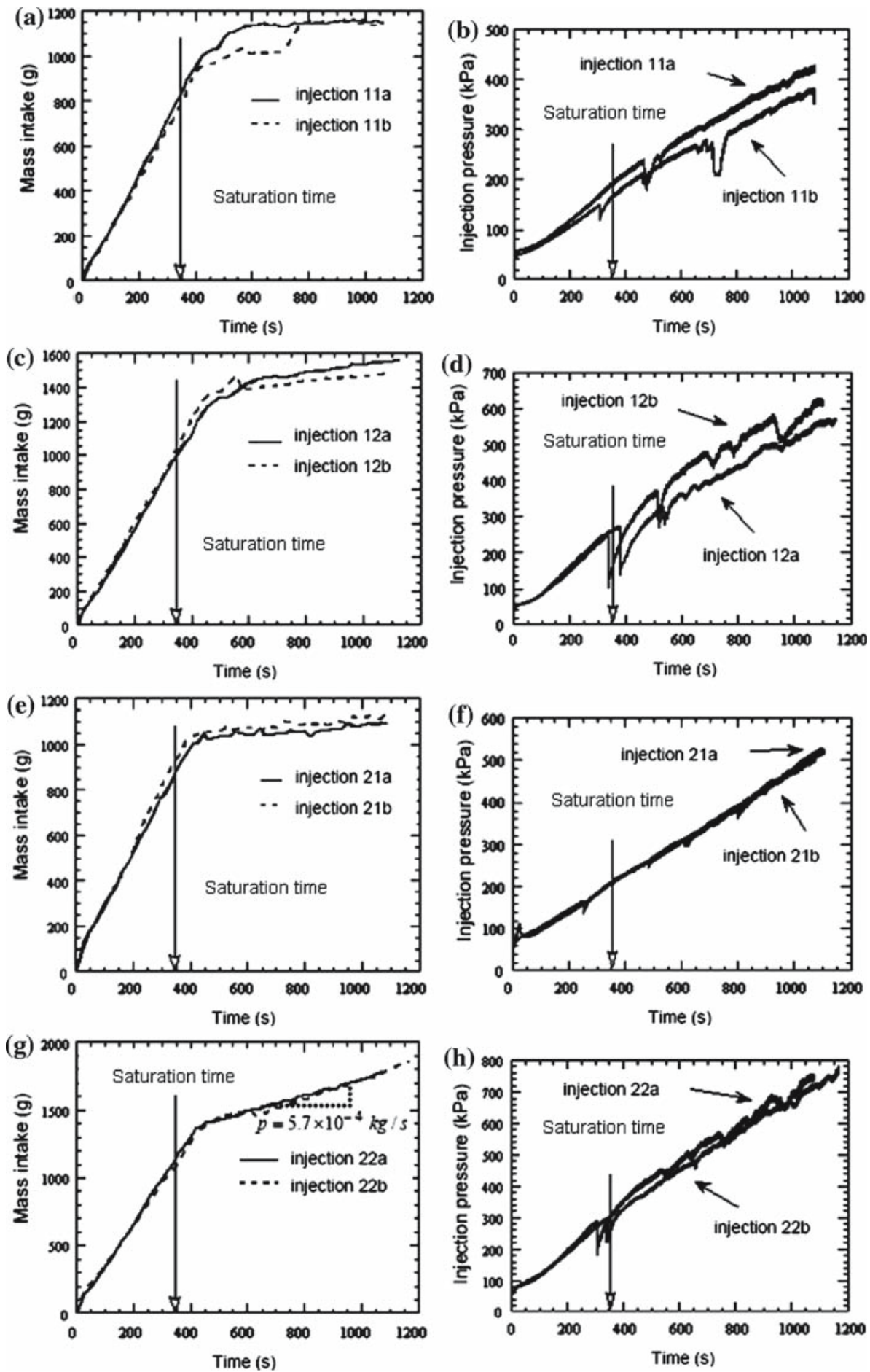

Fig. 3 Mass intake of the sand column and injection pressure vs. time 
Concerning the grout composition, the greater the $C / W$ ratio the higher the injection pressure required to maintain a constant inflow rate. This is noticeable in Fig. 3 by comparing curves (b)-(d) or (f)-(h). The same conclusion holds for the weight gain of the sand sample which is more important for high $C / W$ ratios (comparison of Fig. 3a-c, e-g). Concerning the medium characteristics, though only dense sands are tested, we noticed slightly higher pressure for the samples having the highest density at a given $C / W$ ratio (comparison of Fig. 3b-f, d-h). Note that the porosity should be higher for low density, which would explain some difference. The mass intake is quite similar for both of the tested media when $C / W=0.28$ (Fig. 3a, e). Nevertheless, the slope of the mass evolution curve after saturation is higher in Fig. $3 \mathrm{~g}$ than in Fig. 3c (caution: different scales) indicating that the mass intake rate increases with the initial medium density for a given $C / W$ ratio.

To sum up, the $C / W$ ratio and the initial density of the medium have both an impact on the filtration process which is more important for large $C / W$ ratios and for high densities. Although Fig. 3a, e are close, the corresponding pressure illustrated in Fig. 3b,f are quite different. Even if the filtration effects seem to be insignificant in term of mass intake of the column, they can lead to large pore pressure variations. Note that at the saturation time, the injection pressure of Fig. 3b, $\mathrm{f}$ are almost the same.

\section{Determination of the filtration parameters}

The model presented in Sect. 2 depends on two filtration parameters: $\lambda$ and $\beta$. These are determined using the injection tests presented above and the analytical solution of the mathematical model in a simplified one-dimensional case. This procedure is subject to some assumptions and can be applied only once the saturation time is reached. First, only the advection part is considered in the transport equation, i.e., without dispersion effects. Second, we assume that the fluid phase viscosity remains constant after the saturation and that the density effects are negligible.

Under these conditions, the following analytical solution of the problem

$$
\begin{gathered}
c(x, t)=c_{m} u\left(t-\frac{x \phi}{q}\right) \exp (-\lambda x) ; \quad u\left(t-\frac{x \phi}{q}\right)= \begin{cases}0 & \text { if } t<x \phi / q \\
1 & \text { if } t \geq x \phi / q\end{cases} \\
\chi(x, t)=\frac{\lambda q c_{m}}{\phi}\left(t-\frac{x \phi}{q}\right) \exp (-\lambda x), \quad \text { if } t \geq x \phi / q,
\end{gathered}
$$

is calculated in terms of transported and filtrated component concentrations. The boundary condition, $c(0, t)=c_{m}$, is used in the determination of (8). To compute the integration constant in (9), it is assumed that the concentration of the filtrated particles vanishes at the grout front position, given by the equation: $t=x \phi / q$. Obviously, the concentration of the filtrated particles is also zero when $t<x \phi / q$ since, at this time, the injected fluid has not reached the location where the solution is searched.

Then, based on the expressions obtained above, the analytical mass intake of the sample is deduced from the equation of state of the fluid phase. The latter is assumed to read:

$$
\rho^{f}(\mathbf{x}, t)=\rho_{0}^{f}\left[1+\beta_{c}\left(c-c_{0}\right)+\beta_{\chi}\left(\chi-\chi_{0}\right)\right], \quad(\mathbf{x}, t) \in \Omega \times J .
$$

In (10), the subscript 0 indicates that the quantity under consideration is taken at the initial time, $t=0 . \beta_{c}$ and $\beta_{\chi}$ denote the coefficients of concentration that introduce the effect of the change of density as a result of a change in concentration of the transported suspension and the filtrated particles, respectively. Equation (10) implicitly means that the concentration of 
the filtrated particles is expressed as a number per pore volume. In other words, the filtrated particles remain in the fluid phase in a trapped state (Sharma and Yortsos 1987), and they are not considered to be attached to the skeleton. This assumption enables to keep a constant porosity in calculations and, thus, a constant volume of the fluid phase. Then, the analytical mass intake is easily computed as long as the average density of the fluid phase is known. The latter is calculated by using (8) and (9), and by integrating (10) over the considered one-dimensional domain $[0, l]$. Note that the integration over the whole domain is possible for $t>x l / q$, i.e., after the saturation. The total fluid mass is deduced as a function of time, and the mass difference $(\delta m)$ between two time steps $(\delta t)$ is written:

$$
\delta m=\phi V_{\mathrm{col}}\left(1-\frac{\rho_{w}}{\rho_{\chi}}\right) \frac{q c_{m}}{\phi l}[1-\exp (-\lambda l)] \delta t
$$

$\rho_{w}$ and $\rho_{\chi}$ are the densities of the water and the filtrated particles, respectively. $\phi V_{\text {col }}$ denotes the volume of the fluid phase. $\lambda$ is the only unknown of (11) which is a linear function of time. As noticed during the injection tests, the mass of the sample after the saturation also varies linearly with time (see Fig. 3a, c, e, g). Consequently, the parameter $\lambda$ can be determined from (11) and the experimental mass intake rate $(\Delta m / \Delta t)$ after saturation, i.e., when the mass intake is only influenced by filtration.

To determine the second filtration parameter, the Darcy law is integrated over $[0, l]$ respecting the condition $t>x l / q$. The following equation:

$$
\begin{aligned}
\int_{0}^{l}(1+\beta \chi) \mathrm{d} x= & l+\frac{\beta q c_{m}}{\phi}[1-\exp (-\lambda l)] t \\
& +\beta c_{m}\left[l \exp (-\lambda l)+\frac{\exp (-\lambda l)-1}{\lambda}\right] \\
= & \frac{-K_{0}(\varphi(l)-\varphi(0))}{\mu q}
\end{aligned}
$$

which depends on $\beta$ and $\lambda$ is obtained. (12) is a linear function of time, and, as far as $\lambda$ is computed first, $\beta$ can be estimated using the experimental slope of the curve

$$
K_{0}(\varphi(l)-\varphi(0)) / \mu q=f(t) .
$$

$\varphi$ is the total hydraulic head.

\section{Simulation of injection tests}

A numerical algorithm is used to simulate the injection tests. Both viscosity and density effects are considered. The algorithm is based on several methods that enable overcoming the challenges encountered when solving flow and transport in porous media equations (Frolkovic and De Shepper 2001; Ackerer et al. 1999; Kolditz et al. 1998; Oldenburg and Pruess 1995; Pinder and Cooper 1970; Simpson and Clement 2003, or Voss and Souza 1987).

For a sake of conciseness, the numerical algorithm is explained by using the following formulation

$$
\mathbf{L}(\mathbf{u})=\mathbf{m}^{T} \frac{\partial\left(\mathbf{g}^{T} \mathbf{u}\right)}{\partial t}+\nabla \cdot \mathbf{f}-b=0, \quad(\mathbf{x}, t) \in \Omega \times J
$$


of the coupled set of partial differential equations. Appropriate boundary conditions along $\partial \Omega$ and an initial condition on $\Omega \bigcup \partial \Omega$ are required to solve (14). The following definitions

$$
\begin{gathered}
\mathbf{u}=\left\{\begin{array}{l}
p \\
c \\
\chi
\end{array}\right\}, \quad \mathbf{g}=\left\{\begin{array}{l}
0 \\
1 \\
1
\end{array}\right\}, \quad \mathbf{m}=\left\{\begin{array}{l}
0 \\
\phi \\
\phi
\end{array}\right\}, \quad \mathbf{f}=\left\{\begin{array}{c}
\mathbf{q} \\
c \mathbf{q}-\mathbf{D} \nabla c \\
0
\end{array}\right\} \\
\mathbf{b}=\left\{\begin{array}{c}
0 \\
-\lambda|\mathbf{q}| c \\
\lambda|\mathbf{q}| c
\end{array}\right\},
\end{gathered}
$$

are used in (14). The problem is discretized in time by finite differences using the classical trapezoidal rule (or $\theta$-rule) and in space by the finite element method (FEM). The $\theta$-rule applied to (14) yields

$$
\begin{aligned}
\mathbf{m}^{T} \frac{\left(\mathbf{g}^{T} \mathbf{u}\right)^{n+1}-\left(\mathbf{g}^{T} \mathbf{u}\right)^{n}}{\Delta t}= & -\left[\theta \nabla \cdot(\mathbf{f})^{n+1}+(1-\theta) \nabla \cdot(\mathbf{f})^{n}\right] \\
& +\theta \mathbf{b}^{n+1}+(1-\theta) \mathbf{b}^{n},
\end{aligned}
$$

where $n$ represents the time level and $\Delta t$ is the time step increment. The streamline upwind Petrov/Galerkin (SUPG) method (Brooks and Hughes 1982) is used in the spatial discretization of the transport equation to avoid numerical diffusion and oscillations due to advection dominance. Its implementation is checked in Chupin et al. (2004). The discrete system that ensued from the FEM formulation can be written in the general form

$$
\mathbf{A}\left(\mathbf{U}^{n+1}\right) \mathbf{U}^{n+1}-\mathbf{F}^{n+1}=0, \mathbf{U}^{n+1}=\left\{\begin{array}{l}
\mathbf{p}^{n+1} \\
\mathbf{c}^{n+1} \\
\chi^{n+1}
\end{array}\right\},
$$

where $\mathbf{U}^{n+1}$ contains the discrete state variables at time $n+1$ and $\mathbf{A}$ is the coefficient matrix of the discretized system. (17) is a nonlinear system that can be solved either by standard nonlinear methods (e.g., Newton-Raphson method) or in sequences with an outer iteration. The second choice is selected, and the Gauss-Seidel type algorithm (Langtangen 1999) for systems of nonlinear PDE's is retained. It consists in solving the initial system within an iterative procedure for each state variable separately, leading to a decoupled resolution. For example, if $k$ denotes the iterative index, the flow equation is first solved with respect to $\mathbf{p}^{k, n+1}$ considering the other state variables at the previous iteration, $k-1$. The system relative to the flow equation reads:

$$
\left[\begin{array}{llll}
\mathbf{A}_{p p} & \mathbf{A}_{p c} & \mathbf{A}_{p \chi}
\end{array}\right]_{\mathbf{p}^{k}, \mathbf{c}^{k-1}, \chi^{k-1}}^{n+1}\left\{\begin{array}{c}
\mathbf{p}^{k} \\
\mathbf{c}^{k-1} \\
\chi^{k-1}
\end{array}\right\}^{n+1}=\left\{\mathbf{F}_{p}\right\}^{n+1}
$$

The following expressions

$$
\begin{aligned}
& \mathbf{A}_{p p}=\int_{\Omega} \nabla \mathbf{W}^{T}\left(\frac{\mathbf{k}}{\mu^{f}}\right) \nabla \mathbf{N} \mathrm{d} \Omega, \quad \mathbf{A}_{p c}=0, \quad \mathbf{A}_{p \chi}=0, \\
& \mathbf{F}_{p}=\int_{\Omega} \nabla \mathbf{W}^{T}\left(\frac{\mathbf{k}}{\mu^{f}}\right) \rho^{f} g \mathrm{~d} \Omega+\text { Boundary Conditions, }
\end{aligned}
$$

are used in (18) which is a simple system in the present case. More complex systems can be solved by the numerical algorithm which is designed to also handle multidimensional 


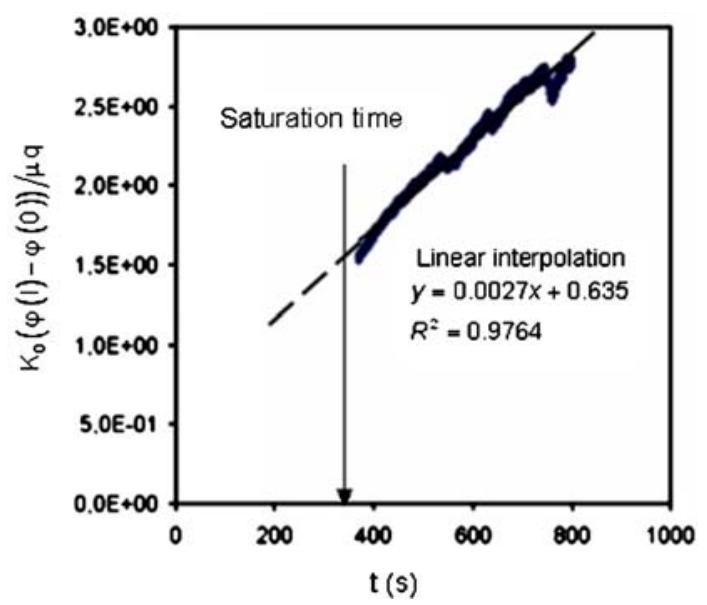

Fig. 4 Evolution of $\left(k_{0}(\varphi(l)-\varphi(0)) / \mu q\right)$ vs. time for Injection22

problems where, for example, $\mathbf{A}_{p c}$ and $\mathbf{A}_{p \chi}$ are not equal to zero. In (20), Boundary Conditions include both Neumann or Robin types, and $\mathbf{W}$ is the FEM weighting function. Once the solution of (18) is known at iteration $k$, the transport and the filtration equations are solved with respect to $\mathbf{c}^{k, n+1}$ and $\chi^{k, n+1}$, respectively, by using the most recently computed state variables. The nonlinearities remaining at the decoupled level are handled with the Picard method. Iterations of the Gauss-Seidel method stop when a convergence criterion is fulfilled. According to Darcy's law, the velocity is deduced from the pressure gradient. To avoid inconsistency in the velocity approximation (Diersch and Kolditz 2002), a smoothing technique, namely the Moving Least-Squares (MLS) technique, is used to derive the velocity field. This technique consists of fitting a linear or a quadratic polynomial to the discrete values of a finite-element field.

Injection22 (Table 2), which exhibits the strongest filtration effects among all the tests performed is simulated.

Before running the calculation, the filtration parameters have to be determined. This is done by applying the procedure described in the above section and by using Figs. 3(g), 4, which represents the evolution of (13) in time. The obtained filtration parameter values are:

$$
\lambda=0.1217 \mathrm{~m}^{-1}, \quad \beta=7.57 \times 10^{-3} \mathrm{~m}^{3} / \mathrm{kg} .
$$

For the determination of $\lambda$ and $\beta, \rho_{\chi}$ is chosen equal to the cement grain density $\left(\rho_{\chi}=2,930\right.$ $\left.\mathrm{kg} / \mathrm{m}^{3}\right)$.

Without precise information about the evolution of the viscosity with regard to the grout concentration within the suspension, a linear law

$$
\mu^{f}(\mathbf{x}, t)=\left(\mu_{g}-\mu_{w}\right) \frac{c}{\rho_{g}}+\mu_{w}, \quad(\mathbf{x}, t) \in \Omega \times J,
$$

is assumed. $\mu_{g}$ and $\rho_{g}$ are the viscosity (measured with a rheometer) and the density of the grout, respectively. $\mu_{w}$ is the viscosity of the water. The domain is discretized by 100 elements of equal length and the time step is set to $1 \mathrm{~s}$. The parameter values used in the simulation are summarized in Table 3 . The boundary conditions are as follows:

$$
c(0, t)=\rho_{g}, \quad \frac{\partial c}{\partial x}(l, t)=0, \quad q(0, t)=q_{\text {imp }}, \quad p(l, t)=0 .
$$


Table 3 Parameter set used in the simulation of Injection 22

\begin{tabular}{lll}
\hline Quantity & Notation & Value \\
\hline Porosity & $\phi$ & 0.308 \\
Permeability & $K_{0}$ & $1.75 \times 10^{-11} \mathrm{~m}^{2}$ \\
Density of the grout & $\rho_{\mathrm{g}}$ & $1,410 \mathrm{~kg} / \mathrm{m}^{3}$ \\
Viscosity of the grout & $\mu_{g}$ & $4.83 \times 10^{-3} \mathrm{~Pa} . \mathrm{s}$ \\
Imposed specific discharge & $q_{\mathrm{imp}}$ & $7.5 \times 10^{-4} \mathrm{~m} / \mathrm{s}$ \\
$\begin{array}{l}\text { Coefficient of longitudinal dispersion } \\
\text { Coefficient of molecular diffusion }\end{array}$ & $a_{\mathrm{L}}$ & $1.0 \times 10^{-3} \mathrm{~m}$ \\
$\begin{array}{l}\text { Concentration coefficient of transpor- } \\
\text { ted particles }\end{array}$ & $B_{\mathrm{d}}$ & $1.0 \times 10^{-10} \mathrm{~m}$ \\
$\begin{array}{l}\text { Concentration coefficient of filtrated } \\
\text { particles }\end{array}$ & $\beta_{\chi}$ & $2.91 \times 10^{-4}$ \\
\hline
\end{tabular}

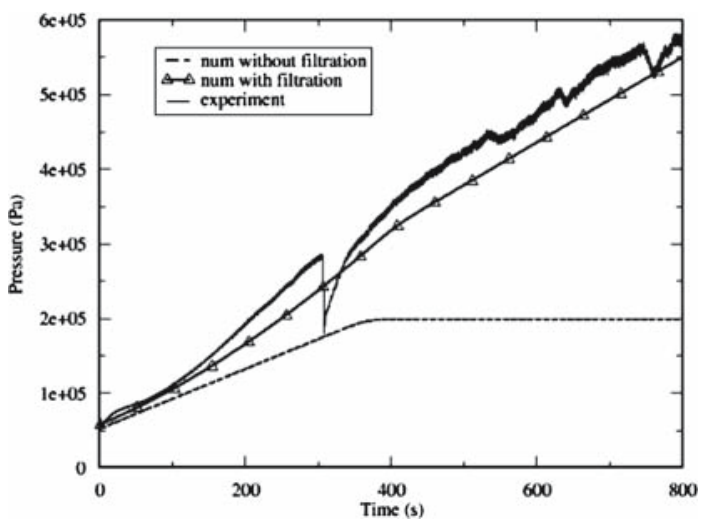

Fig. 5 Evolution of the injection pressure vs. time for Injection22

The entire Injection 22 is simulated. Both pre- and post-saturation phases are studied. Note that the post-saturation phase is only considered in the determination of the filtration parameters. A simulation of Injection22 without including filtration $(\lambda=\beta=0)$ is also performed. These two simulations (with and without filtration) are confronted to the experimental data in terms of injection pressure. The resulting curves are illustrated in Fig. 5. The simulation without filtration is represented by a dotted line and the results including filtration comprise triangular markers. In the first case $(\lambda=\beta=0)$, the injection pressure becomes constant after the saturation, and the simulation curve is far from the experimental measurements plotted as a continuous line, even before the saturation time. However, the simulation with filtration exhibits an injection pressure close to the experimental data during the entire test, and the model improvement is clearly noted. The comparison of the numerical results to the experimental data prove the necessity of considering the phenomenon of filtration in the simulation. The discrepancies observed before the saturation between the experimental data and the numerical solution for $\lambda=\beta=0$ can be mainly explained by the effects of filtration.

Figure 6 plots the variation of the concentration of the transported suspension along the distance to the injection point at different times. The major difference between the two simu- 


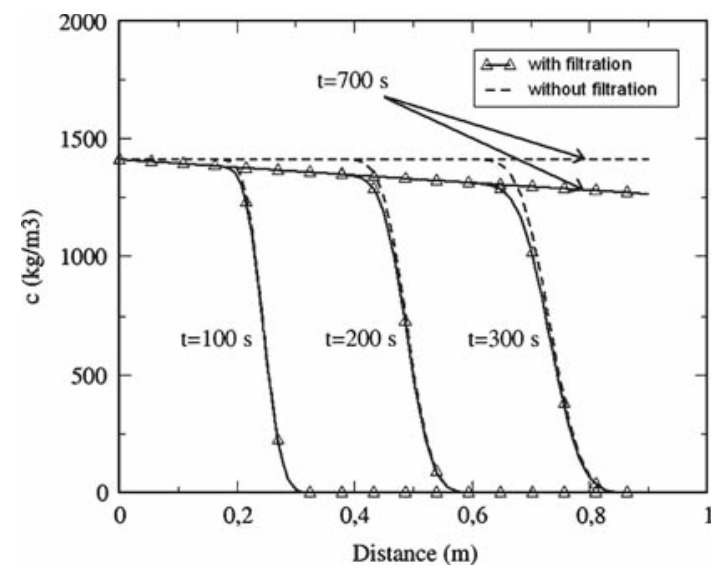

Fig. 6 Concentration at different times of the transported grout against the distance to the injection point. Comparison between the simulations with and without filtration (numerical solution)

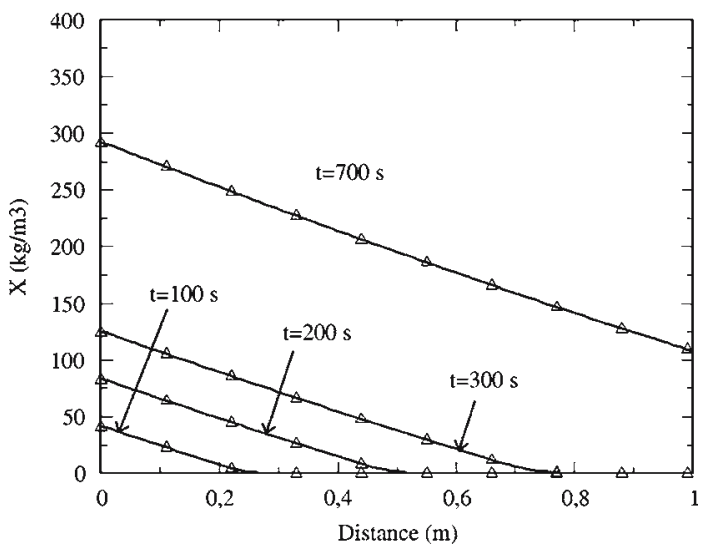

Fig. 7 Concentration at different times of the filtrated particles against the distance to the injection point (numerical solution)

lations (with and without filtration) lies in the concentration profile obtained at the stationary stage $(t=700 \mathrm{~s})$. When this regime is reached and for $\lambda=\beta=0$, the concentration is uniform within the whole sample and is equal to $\rho_{g}$. With filtration, a gradient of concentration is observed. The latter depends essentially on the filtration coefficient, $\lambda$. The higher $\lambda$ the sharper the gradient computed between the two ends of the column. The density of the effluent fluid can also be computed by considering Fig. 6 and the equation of state of the fluid phase. At $t=700 \mathrm{~s}$, this density is approximately equal to $1360 \mathrm{~kg} / \mathrm{m}^{3}$ [the concentration of the filtrated particles is equal to zero outside the sample and it is assumed that $\left.c\left(x=l^{-}, t\right)=c\left(x=l^{+}, t\right)\right]$. This value is not far from the density of the injected grout. The measured density of the effluent suspension is also close to $\rho_{g}$ in the experiments presented in Sect. 3. Moreover, the concentration of the transported suspension computed at time $t=300 \mathrm{~s}$ is still equal to zero at $x=l$. This result is logical since the saturation time which corresponds to the arrival of the grout in the effluent suspension is around $350 \mathrm{~s}$. 
Figure 7 represents the concentration of the filtrated particles against the distance to the injection source, displayed at different times. At $t=700 \mathrm{~s}$, this concentration in $x=0$ is approximately equal to $290 \mathrm{~kg} / \mathrm{m}^{3}$ and indicates that the initial permeability of the medium has been divided by a factor of 3.2 during the simulation (the computation of the permeability uses (3)).

\section{Conclusion}

The experiments and simulations presented in this article prove that filtration plays a significant role during one-dimensional injection tests of cement grouts in sand. Experimentally, this is highlighted by measuring the mass intake of the sand sample and the injection pressure. In the whole duration of the tests, both these quantities increase continuously contrary to the case of a flow which does not involve filtration. In the absence of filtration, a plateau in the mass intake and the injection pressure would be expected after the grout has saturated the sand. Note that to avoid nonfiltration effects which could influence the pressure via a modification of the fluid phase properties, the tests were performed in a duration that does not allow the cementation process to take place.

It has also been shown that the higher the cement-to-water ratio of the injected grout the higher the sample mass intake and injection pressure after saturation. The same conclusion holds for the initial density of the sample. Moreover, after the saturation, a small-mass intake of the column (small amount of filtrated particles) can occur in the same time as a high pressure increase. Thus, either the filtration effects are greater on the injection pressure than on the mass intake or an additional phenomenon to the filtration also influences the pore pressure.

A suitable modeling of the injection tests is obtained by using a flow and transport model which includes the filtration and the damage coefficients. A method based on an analytical solution of the model equations was proposed to determine these coefficients. It uses the mass intake and pressure measurements.

Whereas, a simulation without filtration does not match the experimental results, a simulation which relies on the model described above does. In the latter case, the small remaining discrepancy between simulation and measurement may be reduced by using a different viscosity law which would have to be determine from rheological experiments. Moreover, the effect of varying filtration coefficients could be considered.

Acknowledgements The authors would like to thank C. Dano and Z. Yin for their contribution to the experimental tests.

\section{References}

Ackerer, P., Younes, A., Mose, R.: Variable density flow and transport in porous medium: 1. numerical model and verification. Transport Porous Med. 35, 345-373 (1999)

Bear, J.: Dynamics of Fluid in Porous Media. McGraw-Hill, New York (1972)

Bear, J., Bachmat, Y.: Introduction to Modeling of Transport Phenomena in Porous Media. Kluwer Academic Publishers, Dordrecht (1991)

Bedrikovetsky, P., Marchesin, D., Shecaira, F., Souza, A.L., Milanez, P.V., Rezende, E.: Characterization of deep bed filtration system from laboratory pressure drop measurements. J. Petrol. Sci. Eng. 32, 167177 (2001) 
Bouchelaghem, F., Vulliet, L.: Mathematical and numerical filtration-advection-dispersion model of miscible grout propagation in saturated porous media. Int. J. Numer. Anal. Geomech. 25, 1195-1227 (2001a)

Bouchelaghem, F., Vulliet, L.: Real-scale miscible grout injection experiment and performance of advectiondispersion-filtration model. Int. J. Numer. Anal. Geomech. 25, 1149-1173 (2001b)

Brooks, A.N., Hughes, T.J.R.: Streamline Upwind/Petrov-Galerkin formulations for convection dominated flows with particular emphasis on the incompressible Navier-Stokes equations. Comput. Meth. Appl. Mech. Eng. 32, 199-259 (1982)

Chupin, O.: Ecoulement et transport couplés en milieu poreux saturé: application à l'injection des sols. Dissertation, Ecole Centrale de Nantes et Université de Nantes, France (2005)

Chupin, O., Saiyouri, N., Hicher, P.-Y.: Simulation of miscible grout injection in saturated porous media. In: 9th International Symposium on Numerical Models in Geomechanics, Ottawa (Canada), 25-27 August 2004

Dano, C., Hareb, H., Hicher, P.-H.: Characterization of Loire river sand in the small strain domain using new bender-extender elements. In: 16th ASCE Engineering Mechanics Conference, University of Washington, Seattle, 16-18 July 2003

Diersch, H.-J.G., Kolditz, O.: Variable-density flow and transport in porous media: approaches and challenges. Adv. Water Resour. 25, 899-944 (2002)

Dulien, F.A.L.: Porous Media, Fluid Transport and Pore Structure. Academic Press, Inc. (London) Ltd (1979)

Eriksson, M., Stille, H., Andersson, J.: Numerical calculations for grout spread with account for filtration and varying aperture. Tunn. Undergr. Space Technol. 15, 353-364 (2000)

Frolkovic, P., De Schepper, H.: Numerical modelling of convection dominated transport coupled with density driven flow in porous media. Adv. Water Resour. 24, 63-72 (2001)

Hassanizadeh, M., Gray, W.G.: General conservation equations for multi-phase systems: 1. averaging procedure. Adv. Water Resour. 2, 131-144 (1979)

Herzig, J.P., Leclerc, D.M., LeGoff, P.: Flow of suspensions through porous media - application to deep bed filtration. Ind. Eng. Chem. 62(5), 8-35 (1970)

Isawaki, T.: Some notes on sand filtration. J. Am. Water Works Ass. 29, 1591 (1937)

Kolditz, O., Ratke, R., Diersch, H.-J.G., Zielke, W.: Coupled groundwater flow and transport: 1. verification of variable density flow and transport models. Adv. Water Resour. 21(1), 27-46 (1998)

Langtangen, H.P.: Computational Partial Differential Equations: Numerical Methods and Diffpack Programming. Springer-Verlag, Berlin (1999)

McDowell-Boyer, L.M., Hunt, J.R., Sitar, N.: Particle transport through porous media. Water Resour. Res. 22(13), 1901-1921 (1986)

Oldenburg, C.M., Pruess, K.: Dispersive transport dynamics in a strongly coupled groundwater-brine flow system. Water Resour. Res. 31, 289-302 (1995)

Pinder, G.F., Cooper, H.H.: A numerical technique for calculating the transient position of the saltwater front. Water Resour. Res. 6(3), 875-882 (1970)

Reger, S.D., Fogler, H.S.: A network model for deep bed filtration of solid particles and emulsion drops. AIChE J. 34(11), 1761-1772 (1988)

Saada, Z., Canou, J., Dormieux, L., Dupla, J.C., Maghous, S.: Modelling of cement suspension flow in granular porous media. Int. J. Numer. Anal. Meth. Geomechan. 29, 691-711 (2005)

Schwarz, L.G.: Roles of Rheology and Chemical Filtration on Injectability of Microfine Cement Grout. Ph.D. Thesis, Northwestern University, USA, p. 503 (1997)

Simpson, M.J., Clement, T.P.: Theoretical analysis of the worthiness of Henry and Elder problems as benchmarks of density-dependent groundwater flow models. Adv. Water Resour. 26, 17-31 (2003)

Voss, C.I., Souza, W.R.: Variable density flow and solute transport simulation of regional aquifers containing a narrow freshwater-saltwater transition zone. Water Resour. Res. 26, 2097-2106 (1987)

Sharma, M.M., Yortsos, Y.C.: Transport of particulate suspensions in porous media: model formulation. AIChE J. 33, 1636-1643 (1987)

Tarafdar, S., Dey, A., Gupta, B.S.: A multiple state stochastic model for deep bed filtration. Chem. Eng. Technol. 15(1), 44-50 (1992) 